\section{(6) OPEN ACCESS}

\title{
Sclerostin inhibition reverses systemic, periarticular and local bone loss in arthritis
}

\author{
Xiao-Xiang Chen, ${ }^{1,2}$ Wolfgang Baum, ${ }^{1}$ Denise Dwyer, ${ }^{3}$ Michael Stock, ${ }^{1}$ \\ Kay Schwabe, ${ }^{1}$ Hua-Zhu Ke, ${ }^{3}$ Marina Stolina, ${ }^{3}$ Georg Schett, ${ }^{1}$ Aline Bozec ${ }^{1,4}$
}

\begin{abstract}
Handling editor Tore K Kvien
${ }^{1}$ Department of Internal Medicine 3, University of Erlangen-Nuremberg, Erlangen, Germany

${ }^{2}$ Department of Rheumatology, Renji Hospital, Shanghai, China

${ }^{3}$ Amgen Inc., Thousand Oaks, California, USA

${ }^{4}$ Nikolaus Fiebiger Center of Molecular Medicine, University Hospital Erlangen, University of Erlangen-Nuremberg, Erlangen, Germany
\end{abstract}

\section{Correspondence to}

Professor Aline Bozec or Georg Schett, Department of Internal Medicine 3 and Institute of Clinical Immunology, University of Erlangen-Nuremberg, Erlangen 91054, Germany; aline.bozec@uk-erlangen.de or georg.schett@uk-erlangen.de

Accepted 14 April 2013 Published Online First 10 May 2013

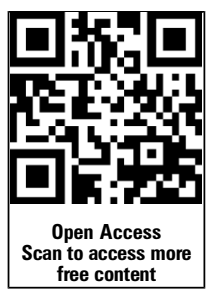

To cite: Chen $X-X$, Baum W, Dwyer D, et al. Ann Rheum Dis 2013;72:1732-1736.

\section{ABSTRACT}

Objective To test whether inhibition of sclerostin by a targeted monoclonal antibody ( $\mathrm{Scl}-\mathrm{Ab}$ ) protects from bone and cartilage damage in inflammatory arthritis. Sclerostin is a potent inhibitor of bone formation and may be responsible for the low level of bone repair in patients with rheumatoid arthritis.

Methods Human tumour necrosis factor transgenic mice (hTNFtg mice) developing inflammatory arthritis and local and bone loss were administered either vehicle, anti-TNF antibody, SCl-Ab, or a combination of both agents. Inflammation, systemic and periarticular bone loss, bone erosion and cartilage damage were evaluated at baseline (week 8) and after 3 weeks of treatment by clinical assessment, micro-CT and histology. Results Scl-Ab did not affect joint swelling or synovitis. Systemic bone loss in the spine and periarticular bone loss in the proximal tibia were completely blocked and partially reversed by inhibition of sclerostin but not by inhibition of TNF. Moreover, Scl-Ab completely arrested the progression of bone erosion in hTNFtg mice and in combination with TNF inhibition even led to significant regression of cortical bone erosions. Protective effects of $\mathrm{Scl}-\mathrm{Ab}$ were also observed for the articular cartilage. Conclusions These data suggest that sclerostin inhibition is a powerful tool to enhance bone repair in inflammatory arthritis.

\section{INTRODUCTION}

Rheumatoid arthritis (RA) leads to generalised bone loss and periarticular bone and cartilage damage. ${ }^{1}$ Bone and cartilage loss contribute to disease burden of RA by destroying joint architecture and increasing fracture risk. ${ }^{2-4}$ Current concepts suggest that inflammation creates an imbalance in bone homeostasis with high-level resorption but low-level bone formation. Whereas therapeutic interventions blocking increased bone resorption, like bisphosphonates and RANKL inhibitors, mitigate local and generalised bone loss in inflammatory arthritis, the effect of enhancing bone formation is poorly studied. This situation is surprising as patients with RA show only limited capacity to repair bone even when treatment with highly effective anti-inflammatory drugs, such as cytokine inhibitors, is commenced. ${ }^{5} 6$

Sclerostin, an osteocyte-specific protein and product of the sclerostin gene (SOST) is a potent suppressor of bone formation. ${ }^{7-9}$ Systemic administration of a targeted sclerostin antibody ( $\mathrm{Scl}-\mathrm{Ab}$ ) increases bone mass in models of ovariectomy-induced osteoporosis, fracture repair and implant healing. ${ }^{10}{ }^{11}$
Furthermore, a phase 1 clinical study has shown that $\mathrm{Scl}-\mathrm{Ab}$ increases bone mass in postmenopausal women. ${ }^{12}$ In RA, variants of the SOST gene have been linked to structural progression of disease. ${ }^{13}$ These data support the concept that Scl-Ab can restore previously lost bone and suggest that such therapeutic approach could be beneficial to reverse the negative consequences of arthritis on bone.

To test this concept, we blocked sclerostin in human tumour necrosis factor transgenic (hTNFtg) mice which spontaneously develop arthritis associated with systemic bone loss, local bone destruction and cartilage damage. Sclerostin blockade was initiated when mice had already developed local and systemic bone loss to permit the assessment for bone repair.

\section{METHODS}

\section{Mice and treatments}

Forty-eight female 8-week-old mice were analysed in two consecutive independent experiments. Eight mice were non-arthritic wild-type littermates (controls). Forty were hTNFtg mice (C57Bl6 background, Tg197 strain): 8 were analysed at the age of 8 weeks (baseline); the other 32 mice were randomised into 4 treatment groups (each $\mathrm{N}=8$ mice): $\operatorname{IgG}(10 \mathrm{mg} / \mathrm{kg}$ by intraperitoneal injection 3 times weekly; negative control), TNF-inhibiting antibody infliximab $(10 \mathrm{mg} / \mathrm{kg}, 3$ times weekly; TNFi) as positive control, Scl-Ab r13c7 (10 mg/kg, 3 times weekly, Scl-Ab) or combination of both antibodies for 3 weeks.

\section{Clinical assessment}

Clinical evaluation was performed weekly, starting at 4 weeks after birth. Arthritis was evaluated in a blinded manner as described previously. ${ }^{14}$

\section{Micro-CT}

The 2nd lumbar vertebral body (for analysis of systemic bone loss) and left proximal tibia metaphysis (for periarticular bone loss) were analysed by micro- CT (GE explore Locus SP Specimen Scanner; GE Healthcare). Images were reconstructed to an isotropic voxel size of $13.2 \mu \mathrm{m}^{3}$, and regions within the vertebral body (central 80\%) and proximal tibia metaphysis $(3 \mathrm{~mm}$, adjacent to the growth plate) were examined. Within these images, trabecular and cortical subregions were outlined using a semiautomated algorithm, and the following parameters were analysed: trabecular bone volume per tissue volume (BV/TV), trabecular thickness (Tb. Th), number (Tb. N) and separation 
(Tb. Sp), connectivity density, using a threshold of $585 \mathrm{mg} / \mathrm{cm}^{3}$. Additionally, bone mineral density (BMD) was generated without thresholding in the trabecular and cortical regions.

\section{Paw histology}

Both hind paws were fixed overnight in 4\% paraformaldehyde, decalcified using EDTA and embedded in paraffin. Sections were stained with hematoxylin-eosin, tartrate-resistant acid phosphatase or toluidine blue for evaluation of synovitis, bone erosions and cartilage (surface area, thickness, proteoglycan content), respectively. Histomorphometric analysis was done by digital analysis system (OsteoMeasure; OsteoMetrics).

\section{Biochemical assays for serum}

Serum collected at the end of a study was used to quantify cytokines (IL-6, MCP1, keratinocyte chemoattractant (KC)) by using multiplex mouse-specific Luminex kits (EMD Millipore, Billerica, Massachusetts, USA)

Data are presented as the mean \pm SEM. For curve comparisons, non-parametric Mann-Whitney test was used. A p value of less than 0.05 was considered significant. For the group comparisons, Unpaired t test with $\mathrm{p}$ values two-tailed were used for the histological analyses.

\section{RESULTS}

\section{$\mathrm{Scl}-\mathrm{Ab}$ does not affect clinical and biochemical}

signs of arthritis

We first investigated whether Scl-Ab affects the signs and symptoms of arthritis. Vehicle-treated mice developed progressively increased joint swelling from week 8 (baseline) to week 11. TNFi arrested progression of arthritis either alone or in combination with Scl-Ab. Single blockade of sclerostin did not show significant reduction of signs and symptoms of arthritis as compared with vehicle treatment (figure 1A). When analysing grip strength, similar results were obtained (figure 1B). Furthermore, $\mathrm{Scl}-\mathrm{Ab}$ did not significantly reduce or increase elevated serum levels of proinflammatory markers, such as interleukin-6, monocyte chemattractant protein-1 and KC. Significant decreases of these markers were observed after TNFi. Body weight gradually decreased during the progression of arthritis in vehicle-treated mice (figure 1C). Surprisingly, Scl-Ab halted weight loss in hTNFtg mice, whereas TNFi permitted physiological increase in body weight between weeks 8 and 11 .

\section{$\mathrm{Scl}-\mathrm{Ab}$ reverses systemic bone loss in arthritis}

We next addressed whether Scl-Ab can influence systemic osteopenia in the lumbar spine. Micro-CT assessment showed a $35 \%$ decrease of bone volume per tissue volume (BV/TV) in vehicletreated hTNFtg mice between week 8 and week 11 (figure 2A). As previously reported, ${ }^{15}$ TNFi monotherapy did not
A.

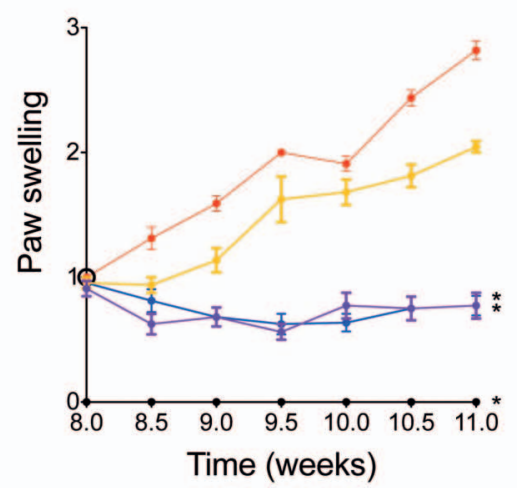

B.
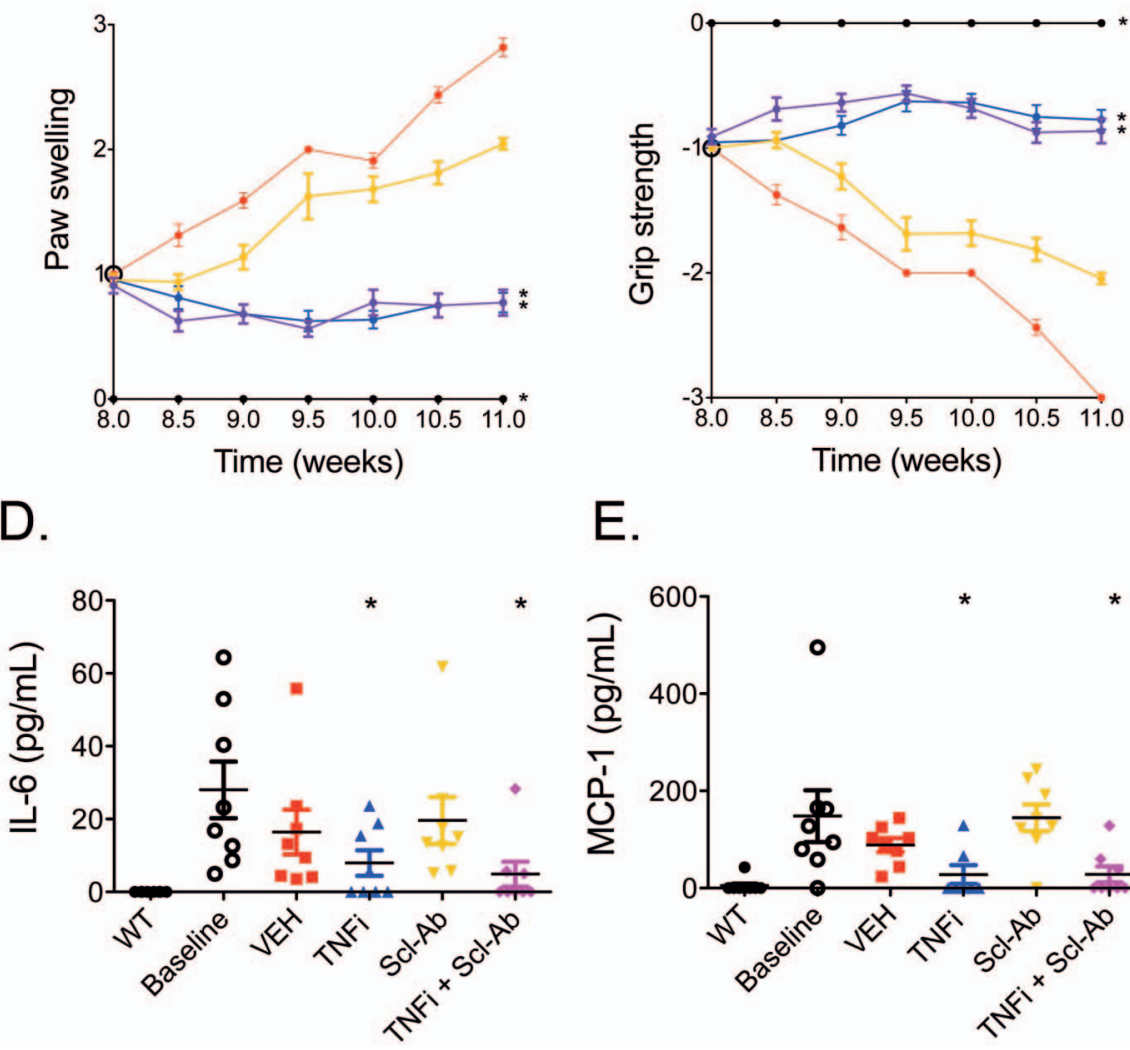

E.

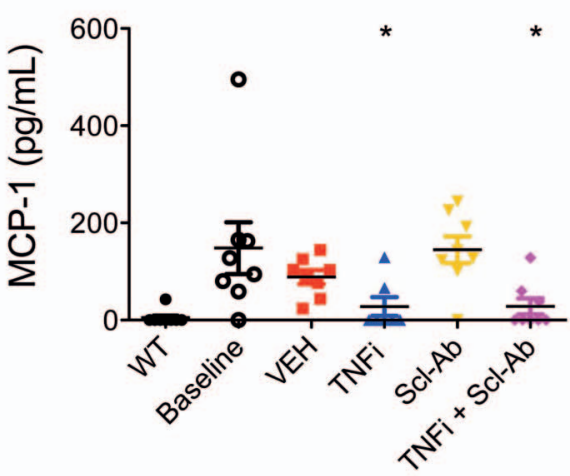

C.

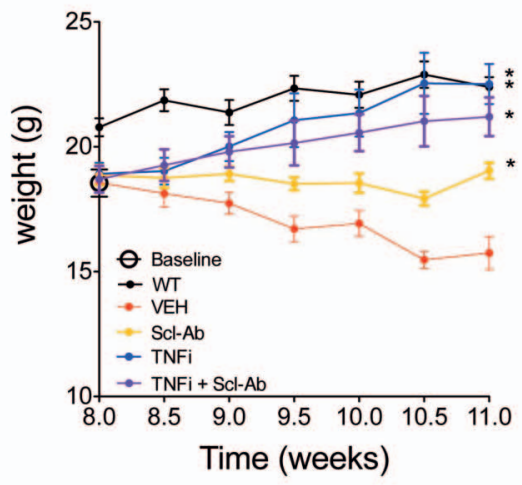

$\mathrm{F}$.

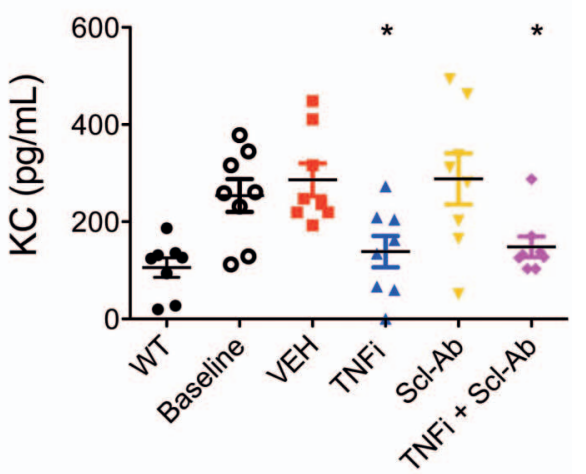

Figure 1 Effects of sclerostin inhibition on clinical and biochemical signs of arthritis. Paw swelling (A), grip strength (B) and body weight (C) assessed in wild-type mice (black) and human tumour necrosis factor transgenic mice at baseline (week 8; black circle) and after treatment with vehicle (VEH, red), tumour necrosis factor inhibitor (TNFi, blue), sclerostin inhibitor (Scl-Ab, yellow) or the combination of both agents (TNFi+Scl-Ab; purple) between week 8 and week $11 .\left(^{*}\right)$ indicates significant $(p<0.05)$ difference to vehicle group. Serum cytokines and chemokines were analysed in the serum of these mice at week 11: (D) interleukin-6 (IL-6), (E) monocyte chemoattractant protein-1 (MCP-1) and (F) keratinocyte chemoattractant $(\mathrm{KC}) .\left(^{*}\right)$ indicates significant $(p<0.05)$ difference to vehicle group. 


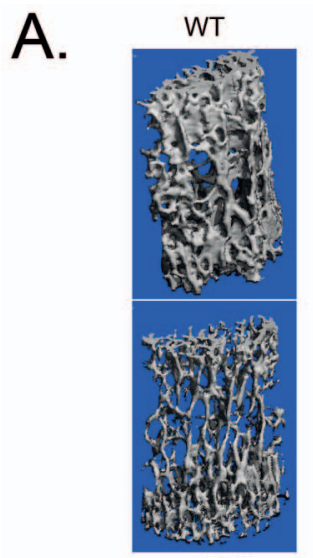

TNFi

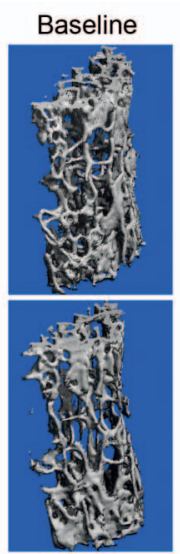

$\mathrm{Scl}-\mathrm{Ab}$

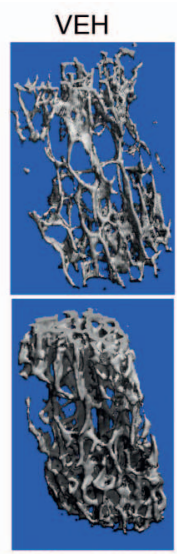

$\mathrm{TNFi}+\mathrm{Scl}-\mathrm{Ab}$
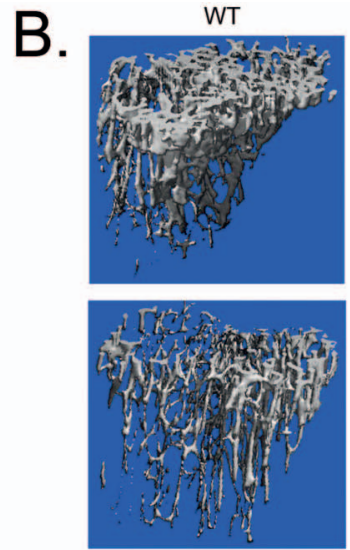

TNFi
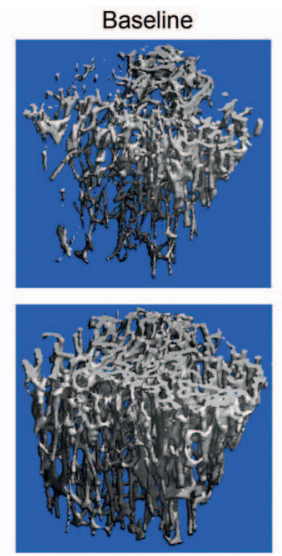

$\mathrm{Scl}-\mathrm{Ab}$
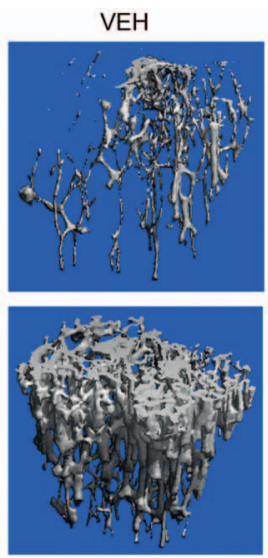

$\mathrm{TNFi}+\mathrm{Scl}-\mathrm{Ab}$
BVITV

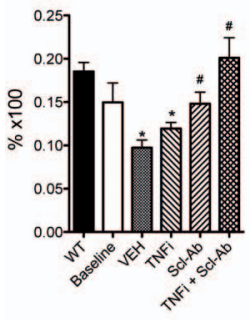

Connectivity Density

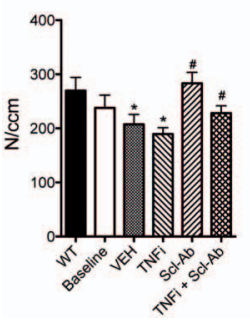

Trabecular Number

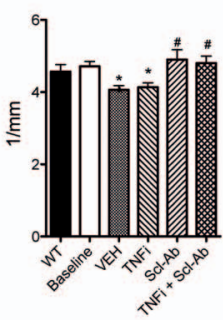

Trabecular BMD

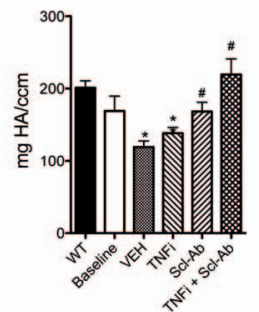

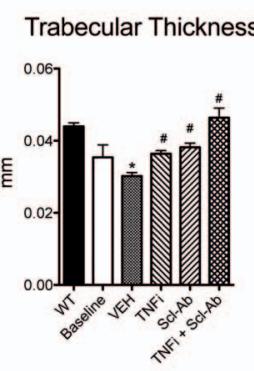

Cortical BMD

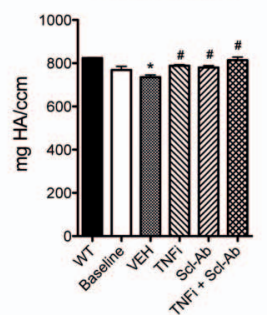

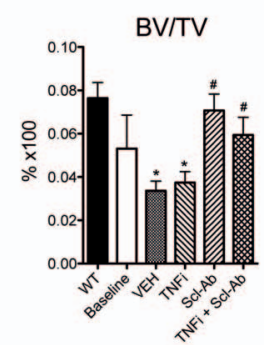

Connectivity Density

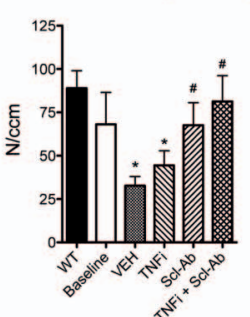

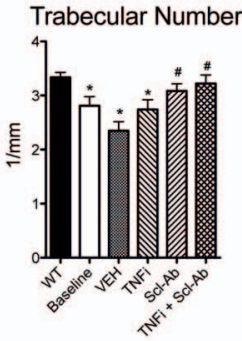

Trabecular BMD

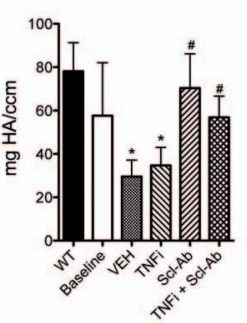

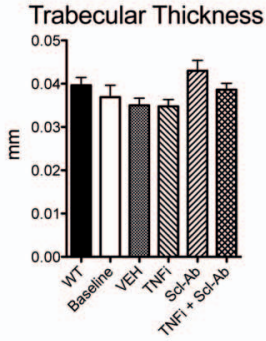

Cortical BMD

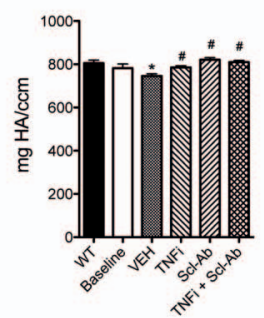

Figure 2 Effects of sclerostin inhibition on systemic and periarticular bone loss in arthritis. Micro CT analysis of the 2nd lumbar vertebral bdoy (A) and proximal tibia metaphysis (B) in wild-type mice (WT) and human tumour necrosis factor transgenic mice at baseline (week 8) and after treatment with vehicle (VEH), tumour necrosis factor inhibitor (TNFi), sclerostin antibody (Scl-Ab) inhibitor or the combination of both agents (TNFi $+\mathrm{Scl}-\mathrm{Ab})$ at week 11. BV/TV, bone volume per tissue volume; BMD, bone mineral density; $\left.{ }^{*}\right)$ indicates significant $(\mathrm{p}<0.05)$ difference to baseline; (\#) indicates significant $(\mathrm{p}<0.05)$ difference to vehicle.

significantly affect systemic bone loss, although individual bone parameters showed slight improvement. By contrast, Scl-Ab either alone $( \pm 0 \%$ compared with baseline) or in combination with TNFi ( $+34 \%$ compared with baseline) completely abolished trabecular and cortical bone loss. Furthermore, the combination of TNFi and Scl-Ab restored vertebral bone to the level observed in non-arthritic wild-type mice (figure 2).

\section{Sclerostin inhibition blocks periarticular bone loss in arthritis}

We next assessed periarticular bone loss in the proximal tibia. $\mathrm{BV} / \mathrm{TV}$ decreased by $38 \%$ between baseline and follow-up in vehicle-treated mice. TNFi did not inhibit periarticular bone loss ($31 \%$ compared with baseline), whereas Scl-Ab achieved complete protection either as monotherapy ( $+32 \%$ compared with baseline) or combined with TNFi (+11\% compared with baseline). Both trabecular and cortical BMD increased in response to Scl-Ab.

\section{Repair of arthritic bone erosions upon combined blockade} of sclerostin and TNF

We then measured bone erosions in the paws of hTNFtg mice receiving the aforementioned treatments. Synovitis progressed from baseline (week 8) to follow-up (week 11). Only TNFi containing treatments did mitigate synovitis, whereas Scl-Ab did not show any anti-inflammatory effect. Bone erosions also progressed significantly from baseline to follow-up. TNFi and $\mathrm{Scl}-\mathrm{Ab}$ did arrest their progression, whereas the combination of TNFi and Scl-Ab suppressed bone erosion to a level which was significantly lower than at baseline. Furthermore, TNFi and $\mathrm{Scl}-\mathrm{Ab}$ halted the significant increase in osteoclast numbers from baseline to follow-up, and combination treatment even significantly suppressed them below baseline levels. Conversely, periarticular osteoblast number was generally low (vehicle: $\mathrm{N}=1.25$ \pm 0.67 /joint) in all groups and only increased significantly after $\mathrm{TNFi} / \mathrm{Scl}-\mathrm{Ab}$ combination treatment $(\mathrm{N}=7.25 \pm 2.46 /$ joint $)$ (figure 3).

\section{Scl-Ab inhibition protects from cartilage damage in arthritis}

Similar to bone erosions, cartilage damage progressed from baseline to follow-up showing significant decreases in cartilage area, cartilage thickness and proteoglycan-rich cartilage. TNFi arrested progression of cartilage damage to baseline level. Scl-Ab, either applied as monotherapy or in combination with TNFi showed even better results: cartilage area, thickness and proteoglycan 
A.
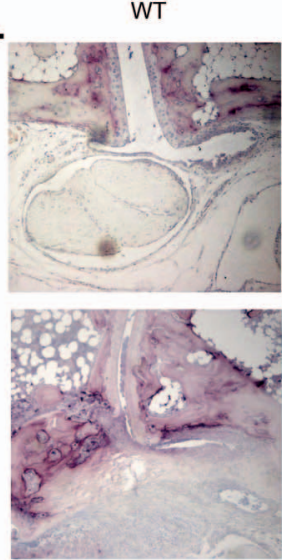

$\mathrm{TNFi}$

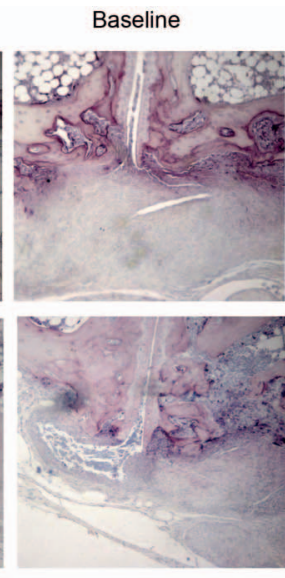

$\mathrm{Scl}-\mathrm{Ab}$
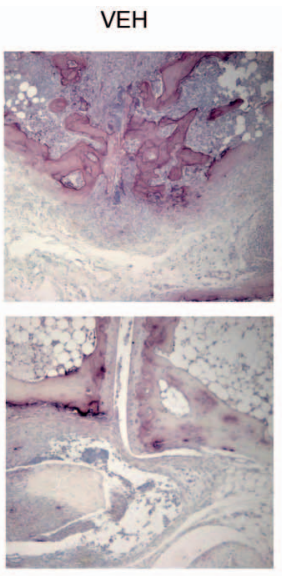

$\mathrm{TNFi}+\mathrm{Scl}-\mathrm{Ab}$
B.
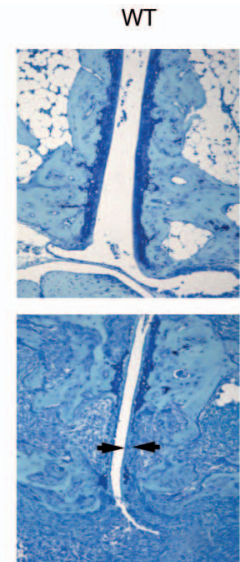

$\mathrm{TNFi}$
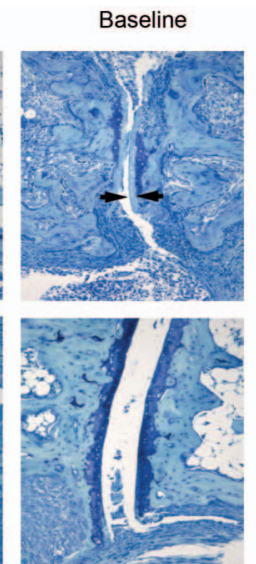

$\mathrm{Scl}-\mathrm{Ab}$
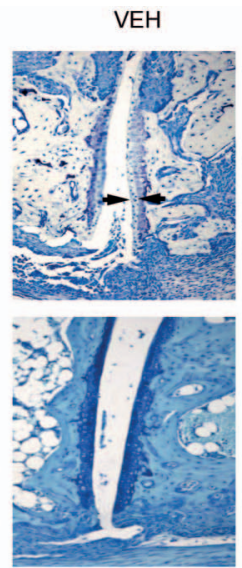

$\mathrm{TNFi}+\mathrm{Scl}-\mathrm{Ab}$

C.
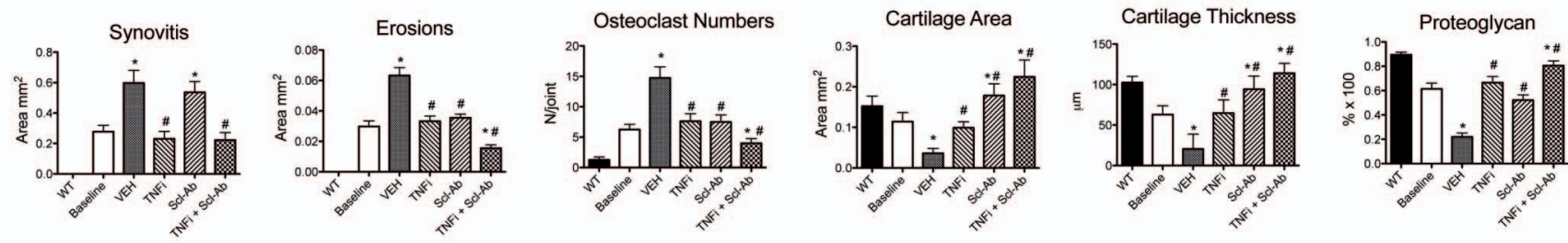

Figure 3 Effects of sclerostin inhibition on arthritic bone erosion and cartilage damage. Tartrate-resistant acid phosphatase staining (A) and toluidine blue staining (B) of hind paws of wild-type mice (WT) and human tumour necrosis factor transgenic mice at baseline (week 8) and after treatment with vehicle (VEH), tumour necrosis factor inhibitor (TNFi), sclerostin antibody (Scl-Ab) inhibitor or the combination of both agents (TNFi $+\mathrm{Scl}-\mathrm{Ab}$ ) at week 11. Purple colour: osteoclasts; dark blue: proteoglycan- rich cartilage; (C) Quantification by histomorphometry showing the area of synovitis in the tarsal joints, the area of bone erosions in the tarsal joints, the number of osteoclasts in the talonavicular joint and cartilage area, thickness and proteoglycan content $\left(\% \times 100\right.$ proteoglycan-rich cartilage) in the talonavicular joint; $\left(^{*}\right)$ indicates significant $(\mathrm{p}<0.05)$ difference to baseline; (\#) indicates significant $(p<0.05)$ difference to vehicle.

content were significantly higher than at baseline level suggesting $\mathrm{Scl}-\mathrm{Ab}$ promoted the repair of articular cartilage (figure 3).

\section{DISCUSSION}

Our study shows that fostering bone formation by Scl-Ab reverses systemic, periarticular and local bone loss in inflammatory arthritis. Scl-Ab also improves articular cartilage damage suggesting it as powerful tool to protect bone and cartilage from the detrimental effect of inflammation and to induce repair of damaged bone and cartilage.

Treatment was initiated at a late stage of arthritis when significant bone and cartilage loss has already occurred. We thereby wanted to mimic the clinical situation of established RA. Bone and cartilage repair is considered an unmet need in patients with RA, since even the most potent anti-inflammatory therapies to date do not reverse bone loss and articular damage. ${ }^{5}{ }^{6} \mathrm{Scl}-\mathrm{Ab}$, but not TNFi, reversed systemic osteopenia in the spine, as well as periarticular osteopenia in the tibia, suggesting that fostering bone formation may be an effective treatment strategy for trabecular bone loss during inflammation. These findings are remarkable as protection of bone occurred despite no significant reduction of inflammation.

By contrast, TNFi alone was not able to reverse systemic and local trabecular bone loss despite its strong anti-inflammatory potential, a finding, which is consistent with observations in human RA. ${ }^{6}$ Given the high prevalence of osteoporosis and increased fracture risk in RA, reversal of trabecular bone loss is an important therapeutic task. ${ }^{3}$ The mere preservation of BMD in RA may not be sufficient, as bone loss starts early in the disease course and a significant proportion of patients with RA are osteopenic already at disease onset. ${ }^{16} 17$
That repair of local bone and cartilage damage is feasible is reflected by the observation that $\mathrm{Scl}-\mathrm{Ab}$ is highly effective in repairing cortical lesions, cartilage destruction and proteoglycan loss when combined with TNFi. Interestingly, a combination of Scl-Ab with TNFi was necessary to achieve repair, whereas inhibition of either Scl-Ab or TNFi alone only blocked progression of bone and cartilage damage but could not reverse it. The effect of TNFi is consistent with observations in human RA showing arrest of progression of bone erosions but only limited repair after TNFi treatment. ${ }^{5}$ These observations suggest that repair of existing bone/cartilage lesions can only be achieved when combining effective anti-inflammatory treatment, like $\mathrm{TNFi}$, with strong bone anabolic agents, such as Scl-Ab. The observation that $\mathrm{Scl}-\mathrm{Ab}$ effected cartilage repair was unanticipated. Restoration of articular cartilage structure may result from direct effects of $\mathrm{Scl}-\mathrm{Ab}$ on cartilage. Although sclerostin is expressed on chondrocytes, ${ }^{18}$ its role in the cartilage is however poorly defined. Another explanation is that cartilage regeneration essentially depends on effective repair of periarticular and subchondral bone which is facilitated by the anabolic effect of Scl-Ab.

In summary, Scl-Ab treatment increases systemic and periarticular bone mass in arthritis. In combination with TNFi, $\mathrm{Scl}-\mathrm{Ab}$ allowed repair of articular cartilage damage and bone erosion suggesting that such combination treatment could set new standards for joint protection.

Contributors $C X-X, W B, M S, K S$ and DD collected the data. AB, MS, H-ZK and GS designed the study. MS and DD contributed to the data analysis and interpretation. $A B$ and $G S$ wrote the manuscript.

Funding This study was supported by the Deutsche Forschungsgemeinschaft (SPP1468-Immunobone), the Bundesministerium für Bildung und Forschung (BMBF; 
project Ankyloss), the Marie Curie Training Network grant Osteoimmune, the Masterswitch and Euroteam project of the European Union and the IMI funded project BTCure. Part of this work was supported by Amgen, Inc., and UCB Pharma.

Competing interests Denise Dwyer, Marina Stolina and Hua-Zhu Ke, are Amgen, Inc. employees, and own Amgen, Inc. stock and/or stock options.

Provenance and peer review Not commissioned; externally peer reviewed.

Open Access This is an Open Access article distributed in accordance with the Creative Commons Attribution Non Commercial (CC BY-NC 3.0) license, which permits others to distribute, remix, adapt, build upon this work non-commercially, and license their derivative works on different terms, provided the original work is properly cited and the use is non-commercial. See: http://creativecommons.org/ licenses/by-nc/3.0/

\section{REFERENCES}

1 Mclnnes IB, Schett G. The pathogenesisi of rheumatoid arthritis. N Engl J Med 2011;365:2205-19.

2 Dequeker J, Geusens P. Osteoporosis and arthritis. Ann Rheum Dis 1990;49:276-80.

3 Schett G, Saag KG, Bijlsma JW. From bone biology to clinical outcome: state of the art and future perspectives. Ann Rheum Dis 2010;69:1415-19.

4 van den Broek M, Dirven L, de Vries-Bouwstra JK, et al. Rapid radiological progression in the first year of early rheumatoid arthritis is predictive of disability and joint damage progression during 8 years of follow-up. Ann Rheum Dis 2012;71:1530-3.

5 Finzel S, Rech J, Schmidt S, et al. Repair of bone erosions in rheumatoid arthritis treated with tumour necrosis factor inhibitors is based on bone apposition at the base of the erosion. Ann Rheum Dis 2011;70:1587-93.

6 Eekman DA, Vis M, Bultink IE, et al. Stable bone mineral density in lumbar spine and hip in contrast to bone loss in the hands during long-term treatment with infliximab in patients with rheumatoid arthritis. Ann Rheum Dis 2011;70:389-90.
7 Balemans W, Ebeling M, Patel N, et al. Increased bone density in sclerosteosis is due to the deficiency of a novel secreted protein (SOST). Hum Mol Genet 2001;10:537-43

8 Brunkow ME, Gardner JC, Van Ness J, et al. Bone dysplasia sclerosteosis results from loss of the SOST gene product, a novel cystine knot-containing protein. Am J Hum Genet 2001;68:577-89.

9 Papapoulos SE. Targeting sclerostin as potential treatment of osteoporosis. Ann Rheum Dis 2011;70:1119-22.

10 Li X, Ominsky MS, Warmington KS, et al. Sclerostin antibody treatment increases bone formation, bone mass, and bone strength in a rat model of postmenopausal osteoporosis. J Bone Miner Res 2009;24:578-88.

11 Ominsky MS, Li C, Li X, et al. Inhibition of sclerostin by monoclonal antibody enhances bone healing and improves bone density and strength of nonfractured bones. J Bone Miner Res 2011:26:1012-21.

12 Padhi D, Jang G, Stouch B, et al. Single-dose, placebo-controlled, randomized study of AMG 785, a sclerostin monoclonal antibody. J Bone Miner Res 2011;26:19-26.

13 de Rooy DP, Yeremenko NG, Wilson AG, et al. Genetic studies on components of the Wnt signalling pathway and the severity of joint destruction in rheumatoid arthritis. Ann Rheum Dis 2013;72:769-75.

14 Diarra D, Stolina M, Polzer K, et al. Dickkopf-1 is a master regulator of joint remodeling. Nat Med 2007;13:156-63.

15 Schett G, Redlich K, Hayer S, et al, Osteoprotegerin protects against generalized bone loss in tumor necrosis factor-transgenic mice. Arthritis Rheum 2003:48:2042-51.

16 Freeston JE, Garnero P, Wakefield RJ, et al. Urinary type II collagen C-terminal peptide is associated with synovitis and predicts structural bone loss in very early inflammatory arthritis. Ann Rheum Dis 2011;70:331-3.

17 Pye SR, Adams JE, Ward KA, et al. Disease activity and severity in early inflammatory arthritis predict hand cortical bone loss. Rheumatology 2010:49:1943-8

18 Roudier M, Li X, Niu QT, et al. Sclerostin is expressed in articular cartilage but loss or inhibition does not affect cartilage remodeling during aging or following mechanical injury. Arthritis Rheum 2013;65:721-31. 